\title{
The growth of mass and metallicity in bulges and disks: CALIFA perspective
}

\author{
R. M. González Delgado ${ }^{1}$, E. Pérez ${ }^{1}$, R. Cid Fernandes ${ }^{1,2}$, \\ R. García-Benito ${ }^{1}$, A. de Amorim ${ }^{2}$, S. F. Sánchez ${ }^{1}$, B. Husemann ${ }^{3}$, \\ R. López Fernández ${ }^{1}$, C. Cortijo ${ }^{1}$, E. Lacerda ${ }^{2}$, D. Mast ${ }^{1}$ and the \\ CALIFA collaboration \\ ${ }^{1}$ Instituto de Astrofísica de Andalucia, Granada, Spain; ${ }^{2}$ Universidade Federal de Santa \\ Catarina, Florianópolis, Brazil; ${ }^{3}$ Leibniz-Institut fur Astrophysik, Postdam, Germany
}

\begin{abstract}
CALIFA (Calar Alto Legacy Integral Field Area) is a 3D spectroscopic survey of 600 nearby galaxies that we are obtaining with PPaK@3.5m at Calar Alto (Sánchez et al. 2012; Husemann et al. 2012). This pioneer survey is providing valuable clues on how the mass and metallicity grow in the different galactic spatial sub-components ("bulge" and "disk"). Processed through spectral synthesis techniques, CALIFA datacubes allow us to, for the first time, spatially resolve the star formation history of galaxies (Cid Fernandes et al. 2012). The richness of this approach is already evident from the results obtained for the first $\sim 100$ galaxies of the sample (Pérez et al. 2012). We have found that galaxies grow inside-out, and that the growth rate depends on a galaxy's mass. Here, we present the radial variations of physical properties sorting galaxies by their morphological type (Figure 1). We have found a good correlation between the stellar mass surface density, stellar ages and metallicities and the Hubble type, but being the the early type spirals $(\mathrm{Sa}-\mathrm{Sbc})$ the galaxies with strong negative age and metallicity gradient from the bulge to the disk.
\end{abstract}

Keywords. galaxies; stellar populations; structure; evolution
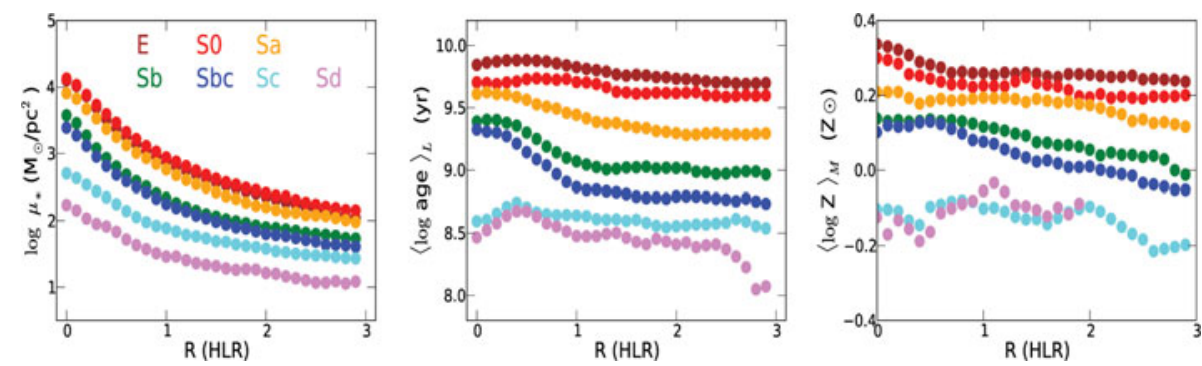

Figure 1. Stellar mass surface density (left), the luminosity-weighted mean stellar age (middle), the mass-weighted mean stellar metallicity (right), radial profiles of 107 galaxies that are stacking by their morphological type.

\section{References}

Cid Fernandes, R., et al. 2012, A\&A submitted

Husemann, B., et al. 2012, A\&SA in press, (arXiv:1210.8150)

Pérez, E., et al. 2012, ApJ submitted

Sánchez, S. F., et al. 2012, A\&A 538, 8 